\title{
AN ANALOGUE OF THE BAIRE CATEGORY THEOREM
}

\author{
PHILIPP HIERONYMI
}

\begin{abstract}
Every definably complete expansion of an ordered field satisfies an analogue of the Baire Category Theorem.
\end{abstract}

\section{INTRODUCTION}

Let $\mathbb{K}$ be an expansion of an ordered field $(K,<,+, \cdot)$. We say $\mathbb{K}$ is definably complete if every bounded subset of $K$ definable in $\mathbb{K}$ has a supremum in $K$. Such structures were first studied by Miller in [7. The main result of this paper is the following definable analogue of the Baire Category Theorem.

Theorem A. Let $\mathbb{K}$ be definably complete. Then there exists no set $Y \subseteq K_{>0} \times K$ definable in $\mathbb{K}$ such that

(i) $Y_{t}$ is nowhere dense for $t \in K_{>0}$,

(ii) $Y_{s} \subseteq Y_{t}$ for $s, t \in K_{>0}$ with $s<t$, and

(iii) $\bigcup_{t \in K>0} Y_{t}=K$,

where $Y_{t}$ denotes the set $\{a \in K:(t, a) \in Y\}$.

Theorem A is a positive answer to a conjecture of Fornasiero and Servi raised in [2, 3. By their work, Theorem A implies that definable versions of many standard facts from real analysis hold in $\mathbb{K}$. Among these are a definable analogue of the Kuratowski-Ulam Theorem, a restricted version of Sard's Theorem and several results in the model theoretic study of Pfaffian functions (see 3, 4, 5, for details).

A short remark about the proof of Theorem A is in order. A definably complete structure does not need to be complete in the topological sense. For this reason the strategy of the classical proof of the Baire Category Theorem to define a sequence of real numbers by recursion is not viable in our setting, as such a sequence might not converge. However, by [2] (see Fact 3 below) it is enough to consider a definable complete $\mathbb{K}$ that defines a closed and discrete set which is mapped by a definable function onto a dense subset of $K$. In such a situation techniques are available that are based on the idea of definable approximation schemes first used by the author in [6. These ideas allow us to replace the use of recursion in the classical proof by an explicit definition of an appropriate sequence.

Notation. In the rest of the paper $\mathbb{K}$ will always be a fixed definably complete expansion of an ordered field $K$. When we say a set is definable in $\mathbb{K}$, we always mean definable with parameters from $\mathbb{K}$. We will use $a, b, c$ for elements of $K$. The letters $d, e$ will always denote elements of a discrete set $D$. Given a subset $X$ of

Date: October 8, 2018.

2000 Mathematics Subject Classification. Primary 03C64; Secondary 54E52.

Some version of this paper will appear in the Journal of Symbolic Logic. 
$K^{n} \times K^{m}$ and $a \in K^{n}$, we denote the set $\{b:(a, b) \in X\}$ by $X_{a}$. We write $\bar{X}$ for the topological closure of $X$ in the usual order topology.

\section{FACTS ABOUt DEFINABLy COMPLETE FIELDS}

In this section we recall several facts about definably complete expansions of ordered fields. For more details and background, see 7 . Let $\mathbb{K}$ be a definably complete expansion of an ordered field.

Fact 1. Let $Y \subseteq K$ be a non-empty closed set definable in $\mathbb{K}$. Then $Y$ contains a minimum and a maximum iff $Y$ is bounded.

Fact 2 ([7, Lemma 1.9]). Let $Y \subseteq K^{2}$ be definable in $\mathbb{K}$ such that $Y_{a}$ is closed and bounded and $Y_{a} \supseteq Y_{b} \neq \emptyset$ for every $a, b \in K$ with $a<b$. Then $\bigcap_{a \in K} Y_{a} \neq \emptyset$.

We say that $\mathbb{K}$ is definably Baire if it satisfies the conclusion of Theorem A. The proof of Theorem A uses the following result of Fornasiero as a starting point.

Fact 3 ([2, Corollary 6.6]). If $\mathbb{K}$ is not definably Baire, then there exists an unbounded, closed and discrete set $D \subseteq K_{\geq 0}$ and a function $f: D \rightarrow K$ such that $f$ is definable in $\mathbb{K}$ and $f(D)$ is dense in $K$.

The strategy for the proof of Theorem A is to establish the following statement: A definably complete expansion of an order field that defines an unbounded, closed and discrete set which is mapped by a definable function onto a dense set, is definably Baire. Note that there are many instances where we already know Theorem A holds. Since $\mathbb{R}$ is a Baire space, every expansion of the real field is definably Baire. Moreover, any o-minimal expansion of an ordered field is definably Baire. For more examples in this direction and related results for expansions of ordered groups, see Dolich, Miller and Steinhorn [1, 3.5].

\section{Proof of Theorem A}

Let $\mathbb{K}$ be a definably complete expansion of an ordered field $(K,<,+, \cdot)$. Towards a contradiction, we assume that there is an increasing family $\left(Y_{t}\right)_{t \in K_{>0}}$ of definable nowhere dense sets such that $K=\bigcup_{t \in K>0} Y_{t}$. Set $Y_{0}:=\emptyset$. Define $X_{t}:=K \backslash Y_{t}$. Then $X_{t}$ is dense in $K$. By replacing $Y_{t}$ by its topological closure, we can assume that $X_{t}$ is open.

By Fact 3 there is also an unbounded, closed and discrete set $D \subseteq K_{\geq 0}$ definable in $\mathbb{K}$ and a map $f: D \rightarrow K$ definable in $\mathbb{K}$ such that the image of $D$ under $f$ is dense in $K$. Since $D$ is cofinal in $K_{>0}$,

$$
K=\bigcup_{d \in D} Y_{d}
$$

Let $\beta: K \rightarrow D \cup\{0\}$ be the function that maps $c$ to the largest $d \in D \cup\{0\}$ such that $c \in X_{d}$. Note that $\beta$ is unbounded. Further let $\gamma: K \rightarrow[0,1]$ map $c \in K$ to the supremum of the set of elements $b$ in $(0,1)$ such that $(c-2 b, c) \subseteq X_{\beta(c)}$. Since $X_{\beta(c)}$ is open, $\gamma(c)>0$. We will write $I_{c}$ for the open interval $(c-\gamma(c), c)$. We will use the following properties of $\beta$ and $\gamma$.

Lemma 4. Let $c \in K$. Then

$$
\emptyset \neq \overline{I_{c}} \subseteq X_{\beta(c)} .
$$


Definition 5. Let $c \in K$. Define $S_{c} \subseteq D$ as the set

$$
\{d \in D: f(d)>c \wedge \forall e \in D e<d \rightarrow(f(e)<c \vee f(d)<f(e))\} .
$$

Moreover, let $S_{c}^{\beta} \subseteq D$ be

$$
\left\{d \in S_{c}: \forall e \in D\left(e<d \wedge e \in S_{c}\right) \rightarrow \beta(f(e))<\beta(f(d))\right\} .
$$

The elements of the set $S_{c}$ can be interpreted as the set of best approximations of $c$ from the right. Compare this to the approximation arguments used in [6. Note that $S_{c}$ is always unbounded, because it does not contain a maximum. The set $S_{c}^{\beta}$ is always non-empty for every $c \in K$, since it contains the minimum of $S_{c}$. But a priori there is no reason why $S_{c}^{\beta}$ should be unbounded. In fact, it might even be finite for some $c \in K$. The advantage of $S_{c}^{\beta}$ over $S_{c}$ is that the composition $\beta \circ f$ is strictly increasing on $S_{c}^{\beta}$.

Definition 6. Let $\delta: K \rightarrow D$ be the function that maps $c$ to the largest $d \in S_{c}^{\beta}$ such that for all $e_{1}, e_{2} \in S_{c}^{\beta}$ with $e_{1}<e_{2} \leq d$

$$
f\left(e_{2}\right) \in I_{f\left(e_{1}\right)} \text {. }
$$

Define $J_{c} \subseteq K$ by

$$
J_{c}:=\bigcap_{e \in S_{c}^{\beta} \cap[0, \delta(c)]} I_{f(e)} .
$$

Lemma 7. The function $\delta$ is well-defined.

Proof. Let $c \in K$. Towards a contradiction, suppose that $\delta(c)$ is not defined. Then $S_{c}^{\beta}$ is unbounded by Fact 1 and for all $e_{1}, e_{2} \in S_{c}^{\beta}$ with $e_{1}<e_{2}$

$$
f\left(e_{2}\right) \in I_{f\left(e_{1}\right)} \text {. }
$$

Then for every $e \in S_{c}^{\beta}$ the set

$$
\bigcap_{e_{1} \in S_{c}^{\beta}, e_{1} \leq e} \overline{I_{f\left(e_{1}\right)}} \subseteq X_{\beta(f(e))}
$$

contains $f(e)$, and hence is non-empty and closed. By Fact 2 and Lemma 4

$$
\emptyset \neq \bigcap_{e \in S_{c}^{\beta}} \overline{I_{f(e)}} \subseteq \bigcap_{e \in S_{c}^{\beta}} X_{\beta(f(e))}
$$

Since $S_{c}^{\beta}$ is unbounded and $\beta \circ f$ is strictly increasing on $S_{c}^{\beta}$, the set $\{\beta(f(e)): e \in$ $\left.S_{c}^{\beta}\right\}$ does not contain a maximum. Thus by Fact 1 it is unbounded. Hence it is cofinal in $D$ and

$$
\bigcap_{e \in S_{c}^{\beta}} X_{\beta(f(e))}=\bigcap_{d \in D} X_{d}
$$

This is a contradiction, since $\bigcap_{d \in D} X_{d}$ is empty.

Lemma 8. Let $c \in K$. Then $J_{c}$ is a non-empty open interval.

Proof. Let $d_{1} \in D$ be the largest element of $S_{c}^{\beta} \cap[0, \delta(c)]$ such that

$$
\bigcap_{e \in S_{c}^{\beta} \cap\left[0, d_{1}\right]} I_{f(e)}
$$

is a non-empty open interval. Such an element exists, since $S_{c}^{\beta}$ is non-empty and $I_{a}$ is a non-empty open interval for every $a \in K$. Towards a contradiction, suppose 
that $d_{1}<\delta(c)$. Let $d_{2} \in D$ be the smallest element in $S_{c}^{\beta} \cap[0, \delta(c)]$ larger than $d_{1}$. Since $d_{2} \leq \delta(c), f\left(d_{2}\right) \in I_{f(e)}$ for all $e \in S_{c}^{\beta}$ with $e<d_{2}$. Hence

$$
I_{f\left(d_{2}\right)} \cap \bigcap_{e \in S_{c}^{\beta} \cap\left[0, d_{1}\right]} I_{f(e)}
$$

is a non-empty open interval. This is a contradiction to the maximality of $d_{1}$. Hence $d_{1}=\delta(c)$.

Note that for every $c \in K$

$$
f(\delta(c)) \in \overline{J_{c}} \subseteq X_{\beta(f(\delta(c)))} .
$$

In order to find a counter-example to the statement $\bigcap_{d \in D} X_{d}=\emptyset$, we will amalgamate sets of the form $S_{c}^{\beta} \cap[0, \delta(c)]$. For this purpose we introduce the following notion of an extension.

Definition 9. For $c_{1}, c_{2} \in K$, we say that $c_{2}$ extends $c_{1}$ if $\delta\left(c_{1}\right)<\delta\left(c_{2}\right)$ and

$$
S_{c_{1}}^{\beta} \cap\left[0, \delta\left(c_{1}\right)\right]=S_{c_{2}}^{\beta} \cap\left[0, \delta\left(c_{1}\right)\right] .
$$

In the following we will construct an unbounded definable subset $E_{0}$ of $D$ such that for all $d, e \in E_{0}$ with $d<e, f(e)$ extends $f(d)$. Given such a set $E_{0}$, we will be able create a contradiction as in the proof of Lemma 7 (see proof of Theorem A below). With that goal in mind, we start by establishing several properties of extensions. First note that being an extension is transitive. If $c_{3}$ extends $c_{2}$ and $c_{2}$ extends $c_{1}$, then $c_{3}$ extends $c_{1}$.

Lemma 10. Let $c_{1}, c_{2} \in K$ be such that $c_{2}$ extends $c_{1}$. Then

(i) $J_{c_{2}} \subseteq J_{c_{1}}$, and

(ii) $\beta\left(f\left(\delta\left(c_{2}\right)\right)\right)>\beta\left(f\left(\delta\left(c_{1}\right)\right)\right)$.

Proof. (i) Since $c_{2}$ extends $c_{1}, S_{c_{1}}^{\beta} \cap\left[0, \delta\left(c_{1}\right)\right] \subseteq S_{c_{2}}^{\beta} \cap\left[0, \delta\left(c_{2}\right)\right]$. Hence $J_{c_{2}} \subseteq J_{c_{1}}$.

(ii) Since $c_{2}$ extends $c_{1}, \delta\left(c_{1}\right) \in S_{c_{2}}^{\beta}$. Since $\beta \circ f$ is strictly increasing on $S_{c_{2}}^{\beta}$, $\beta\left(f\left(\delta\left(c_{2}\right)\right)\right)>\beta\left(f\left(\delta\left(c_{1}\right)\right)\right)$.

Lemma 11. Let $c \in K$ and $d \in D$. If the set

$$
L:=\{f(e): e \in D, e<d, f(e)<c\}
$$

is non-empty, then it contains a maximum.

Proof. Suppose $L$ is non-empty. Then the set

$\left\{e_{1} \in D: f\left(e_{1}\right)<c \wedge \forall e_{2} \in D\left(e_{2}<e_{1}\right) \rightarrow\left(f\left(e_{2}\right)>c \vee f\left(e_{2}\right)<f\left(e_{1}\right)\right)\right\} \cap(0, d)$

is bounded and non-empty. Thus it contains a maximum, say $e_{3}$. By the definition, the image of $e_{3}$ under $f$ is the maximum of $L$.

Proposition 12. Let $c \in K$. Then there exists $d \in D$ such that $f(d)$ extends $c$.

Proof. By Lemma 8 , the set

$$
A:=K_{>c} \cap J_{c}
$$

is a non-empty open interval. We will construct $d, d_{1} \in D$ such that $f\left(d_{1}\right) \in A$, $f(d)$ extends $c$ and $d_{1}$ is the smallest element in $S_{f(d)}^{\beta}$ larger than $\delta(c)$. Because $f\left(d_{1}\right) \in A, d_{1}$ witnesses that $\delta(f(d))>\delta(c)$. 
Since $f(D)$ is dense in $K$, we can define $d_{1} \in D$ as the smallest element in $D$ such that $f\left(d_{1}\right) \in A$ and $\beta\left(f\left(d_{1}\right)\right)>\beta\left(f(\delta(c))\right.$. Since $c<f\left(d_{1}\right)<f(\delta(c))$ and $\delta(c) \in S_{c}$, we have $d_{1}>\delta(c)$. We now choose $d$. By Lemma 11] the set

$$
\left\{f(e): e \in D, e<d_{1}, f(e)<f\left(d_{1}\right)\right\}
$$

has a maximum, say $f\left(d_{2}\right)$ for some $d_{2} \in D$. By density of $f(D)$, we can choose $d \in D$ such that $f(d) \in A \cap\left(f\left(d_{2}\right), f\left(d_{1}\right)\right)$.

Since $c<f(d)<f(\delta(c))$,

$$
S_{c}^{\beta} \cap[0, \delta(c)]=S_{f(d)}^{\beta} \cap[0, \delta(c)] .
$$

It is only left to establish that $\delta(f(d))>\delta(c)$. Since $f\left(d_{1}\right) \in A$, it is enough to show that $d_{1}$ is the smallest element in $S_{f(d)}^{\beta}$ larger than $\delta(c)$. By the choice of $d$, we have that for all $e \in D$ with $e<d_{1}$

$$
c<f(d)<f\left(d_{1}\right)<f(e) \text { or } f(e)<f(d) .
$$

Hence $d_{1} \in S_{f(d)}$. Let $e \in D$ be such that $\delta(c)<e<d_{1}$. We will show that $e \notin S_{f(d)}^{\beta}$. This then directly implies that $d_{1} \in S_{f(d)}^{\beta}$ and that $d_{1}$ is the smallest such element larger than $\delta(c)$. By minimality of $d_{1}$ either $f(e) \notin A$ or $\beta(f(e)) \leq$ $\beta\left(f(\delta(c))\right.$. In both cases we have to check that $e \notin S_{f(d)}^{\beta}$. If $\beta(f(e)) \leq \beta(f(\delta(c))$, then $e \notin S_{f(d)}^{\beta}$ because $\delta(c) \in S_{f(d)}^{\beta}$ and $\beta \circ f$ is strictly increasing on $S_{f(d)}^{\beta}$. Now consider the case that $f(e) \notin A$. Since $A$ is an interval and $f(\delta(c)) \in \bar{A}$, either $f(e)<f\left(d_{1}\right)$ or $f(e) \geq f(\delta(c))$. If $f(e) \geq f(\delta(c))$, then $e \notin S_{f(d)}^{\beta}$ because $\delta(c) \in$ $S_{f(d)}^{\beta}$. If $f(e)<f\left(d_{1}\right)$, then $f(e)<f(d)$ by (3.1). Hence $e \notin S_{f(d)}^{\beta}$. Hence $d_{1}$ is the smallest element in $S_{f(d)}^{\beta}$ larger than $\delta(c)$.

Definition 13. Define $E$ as the set of $e \in D$ such that there is no $d \in D$ with $d<e$ and

$$
S_{f(e)}^{\beta} \cap[0, \delta(f(e))]=S_{f(d)}^{\beta} \cap[0, \delta(f(e))] .
$$

The set $E$ is defined in a way to make sure that if $e \in E, d \in D$ and $f(d)$ extends $f(e)$, then $e<d$.

Lemma 14. Let $c \in K$. The set $\{e \in E: f(e)$ extends $c\}$ is unbounded.

Proof. Let $d_{1} \in D$ be the smallest element in $D$ such that $f\left(d_{1}\right)$ extends $c$. It is easy to see that $d_{1} \in E$. Towards a contradiction, suppose there exists $e \in E$ such that $e$ is the largest element in $E$ such that $f(e)$ extends $c$. By Proposition 12, let $d \in D$ be the smallest element of $D$ such that $f(d)$ extends $f(e)$. Because $e \in E, d>e$. Since $f(e)$ extends $c$, so does $f(d)$. Moreover, since $e \in E$ and $d$ is the smallest element in $D$ such that $f(d)$ extends $f(e), d$ is in $E$ as well. This contradicts the maximality of $e$. Hence the set $\{e \in E: f(e)$ extends $c\}$ is unbounded.

Lemma 15. Let $d_{1}, d_{2}, d_{3} \in D$ be such that $d_{2} \in E$ and $d_{1}<d_{2}<d_{3}$. If $f\left(d_{3}\right)$ extends $f\left(d_{1}\right)$ and $f\left(d_{3}\right)$ extends $f\left(d_{2}\right)$, then $f\left(d_{2}\right)$ extends $f\left(d_{1}\right)$.

Proof. Towards a contradiction, suppose that $\delta\left(f\left(d_{2}\right)\right) \leq \delta\left(\left(f\left(d_{1}\right)\right)\right.$. Since $f\left(d_{3}\right)$ extends both $f\left(d_{1}\right)$ and $f\left(d_{2}\right)$,

$$
S_{f\left(d_{2}\right)}^{\beta} \cap\left[0, \delta\left(f\left(d_{2}\right)\right)\right]=S_{f\left(d_{1}\right)}^{\beta} \cap\left[0, \delta\left(f\left(d_{2}\right)\right)\right] .
$$


This contradicts $d_{2} \in E$. Hence $\delta\left(f\left(d_{2}\right)\right)>\delta\left(f\left(d_{1}\right)\right)$ and

$$
S_{f\left(d_{1}\right)}^{\beta} \cap\left[0, \delta\left(f\left(d_{1}\right)\right)\right]=S_{f\left(d_{2}\right)}^{\beta} \cap\left[0, \delta\left(f\left(d_{1}\right)\right)\right] .
$$

Definition 16. Let $d_{0}$ be the smallest element in $E$. Define $E_{0} \subseteq E$ as the set of elements $d$ of $E$ satisfying the following two properties:

- either $f(d)$ extends $f\left(d_{0}\right)$ or $d=d_{0}$,

- if there are $e_{1}, e_{2} \in E$ such that $d_{0} \leq e_{1}<d, f(d)$ extends $f\left(e_{1}\right)$ and $e_{2}$ is the smallest element in $E$ larger than $e_{1}$ such that $f\left(e_{2}\right)$ extends $f\left(e_{1}\right)$, then either $d=e_{2}$ or $f(d)$ extends $f\left(e_{2}\right)$.

The set $E_{0}$ is definable in $\mathbb{K}$, since both $E$ and the property of being an extension are definable in $\mathbb{K}$.

Lemma 17. Let $d \in E_{0}$. If $e$ is the smallest element in $E$ larger than $d$ such that $f(e)$ extends $f(d)$, then $e \in E_{0}$.

Proof. Since $f(e)$ extends $f(d), f(e)$ extends $f\left(d_{0}\right)$. Let $e_{1}, e_{2} \in E$ be such that $d_{0} \leq e_{1}<e, f(e)$ extends $f\left(e_{1}\right)$ and $e_{2}$ is the smallest element in $E$ larger than $e_{1}$ such that $f\left(e_{2}\right)$ extends $f\left(e_{1}\right)$. If $e_{1}=d$, we get $e_{2}=e$ by minimality of $e$. If $e_{1}<d$, then $f(d)$ extends $f\left(e_{1}\right)$ by Lemma 15 Since $d \in E_{0}$, either $d=e_{2}$ or $f(d)$ extends $f\left(e_{2}\right)$. Thus $f(e)$ extends $f\left(e_{2}\right)$. Hence we can reduce to the case that $e_{1}>d$. Since $f(e)$ extends both $f(d)$ and $f\left(e_{1}\right), f\left(e_{1}\right)$ extends $f(d)$ by Lemma 15 But then $e_{1}=e$ by the minimality of $e$. Hence $e \in E_{0}$.

Proposition 18. Let $d, e \in E_{0}$. If $d<e$, then $f(e)$ extends $f(d)$.

Proof. Consider the set

$$
Z:=\left\{d \in E_{0}: \forall e_{1}, e_{2} \in E_{0}\left(e_{1} \leq d \wedge e_{1}<e_{2}\right) \rightarrow\left(f\left(e_{2}\right) \text { extends } f\left(e_{1}\right)\right)\right\} .
$$

It is enough to show that $Z$ is unbounded. Since $d_{0} \in Z$ by definition of $E_{0}, Z$ is non-empty. For a contradiction, suppose $d_{1} \in D$ is the largest element in $Z$. Let $d_{2}$ be the smallest element in $E$ such that $f\left(d_{2}\right)$ extends $f\left(d_{1}\right)$. By Lemma 17 $d_{2} \in E_{0}$. For every $e \in E_{0}$ with $e>d_{1}$, either $e=d_{2}$ or $f(e)$ extends $f\left(d_{2}\right)$ by definition of $E_{0}$. Hence $d_{2} \in Z$. This contradicts the maximality of $d_{1}$.

Proof of Theorem A. We will show that

$$
\emptyset \neq \bigcap_{d \in E_{0}} \overline{J_{f(d)}} \subseteq \bigcap_{d \in D} X_{d} .
$$

This contradicts the assumption that the family $\left(Y_{d}\right)_{d \in D}$ witnesses that $\mathbb{K}$ is not definably Baire, and hence establishes Theorem A.

By Proposition 18 and Lemma 10, we have for all $d_{1}, d_{2} \in E_{0}$ with $d_{1}<d_{2}$

$$
\overline{J_{f\left(d_{2}\right)}} \subseteq \overline{J_{f\left(d_{1}\right)}} .
$$

By Fact 2 ,

$$
\emptyset \neq \bigcap_{d \in E_{0}} \overline{J_{f(d)}} \subseteq \bigcap_{d \in E_{0}} X_{\beta(f(\delta(f(d)))} .
$$

By Lemma 14 and 17, the set $E_{0}$ has no maximum and hence is unbounded by Fact 1. Hence $\left\{\beta(f(\delta(f(d)))): d \in E_{0}\right\}$ is unbounded as well by Proposition 18 and Lemma 10, Thus the set $\bigcap_{d \in D} X_{d}$ is equal to $\bigcap_{d \in E_{0}} X_{\beta(f(\delta(f(d))))}$ and in particular non-empty. 


\section{REFERENCES}

[1] A. Dolich, C. Miller, C. Steinhorn, Structures having o-minimal open core, Trans. Amer. Math. Soc. (3) 362 (2010) 1371-1411

[2] A. Fornasiero, Definably complete structures are not pseudo-enumerable, Arch. Math. Logic, (5) 50 (2011) 603-615

[3] A. Fornasiero, T. Servi, Definably complete Baire structures, Fund. Math. 209 (2010) 215-241

[4] A. Fornasiero, T. Servi, Pfaffian closure for Definably Complete Baire Structures, Illinois J. Math. to appear

[5] A. Fornasiero, T. Servi, Theorems of the Complement, Proceedings of the Thematic Semester in o-minimality at the Fields Institute of Toronto (Spring 2009) to appear

[6] P. Hieronymi, Defining the set of integers in expansions of the real field by a closed discrete set, Proc. Amer. Math. Soc. 138 (2010) 2163-2168.

[7] C. Miller, Expansions of Dense Linear Orders with the Intermediate Value Property, $J$. Symbolic Logic (4) 66 (2001) 1783-1790

University of Illinois at Urbana-Champaign, Department of Mathematics, 1409 W. Green Street, Urbana, IL 61801, USA

E-mail address: P@hieronymi.de 